\title{
Leptospirose-infecção e forma subclínica em crianças de Salvador, Bahia
}

\author{
Leptospiral infection and subclinical presentation \\ among children in Salvador, Bahia
}

\author{
Hagamenon R. Silva1', José Tavares-Neto², José Carlos Bina² e Roberto Meyer ${ }^{3}$
}

\begin{abstract}
Resumo Os objetivos deste trabalho foram estimar a freqüência da leptospirose e os fatores de risco em crianças de 2 a 15 anos de idade, de ambos sexos, contactantes-domiciliares dos pacientes internados (casosíndices) no Hospital Couto Maia, com diagnóstico de leptospirose (ELISA-IgM positivo). De 148 pessoas, dos domicílios dos 25 casos-índices, selecionados aleatoriamente, 73 (49,3\%) contatos-domiciliares foram submetidos à avaliação clínico-epidemiológica e ao mesmo teste diagnóstico em soros pareados. Os resultados das IgM e Ig G classificaram os 73 contatos-domiciliares em três grupos: A (infecção aguda ou recente), 30 $(41,1 \%)$ casos; $B$ (não-infectados), 34(46,6\%) crianças e $C$ (infecção antiga), nove (12,3\%). Entre os do Grupo $A$, cinco $(16,7 \%)$ apresentaram manifestacóes de resfriado comum. O sexo, o grupo racial, a idade e a presença de cão no domicílio não apresentaram diferenças estatísticas significantes, entre os grupos $A$ e B. Todavia, nas crianças do grupo A predominaram $(p<0,05)$ : contato com água na natureza; irmão caso-índice e menor de 15 anos; sexo feminino e maior de 9 anos de idade e, principalmente, a falta de coleta do lixo nos locais de residência. Em conclusão, a leptospirose em crianças de Salvador é subestimada, porque é oligossintomática ou similar ao resfriado comum.
\end{abstract}

Palavras-chaves: Leptospirose. Criança. Leptospirose infecção. Formas assintomáticas.

Abstract The purposes of this investigation were to identify the frequency and risk factors of leptospiral infection among children aged between 2 and 15 years in Salvador, Bahia, household contacts of patients (index-cases) hospitalized at Couto Maia Hospital due to Leptospira interrogans (ELISA IgM positive). Among 148 household contacts from 25 selected index-cases, clinical and epidemiological data were collected and ELISA IGM and IgG were performed in paired sera (between 20 days) from 73 (49.3\%). This diagnostic method divided the 73 household contacts into three groups: group A (acute or recent leptospiral infection), 30 (41.1\%) children, group B (not- infected) 34 (46.6\%) and group C, (past infection) nine (12.3\%) children. In group A five (16.7\%) had cold syndrome simile. No statistically significant difference was found between groups $A$ and $B$ for sex, race, age and presence of pet dog. However, in group $A$ there was a statistically significant predominance ( $p<$ $0.05 \%)$ of: contact with natural water courses; index-case sibling aged under fifteen years; female sex and over 9 years old; and principally lack of appropiate trash disposal in the residential area. In conclusion, leptospiral infection in children (from 2 to 15 years of age) in Salvador is underestimated, because it is asymptomatic or presents as a cold syndrome simile.

Key-words: Leptospirosis. Children. Leptospiral infection. Asymptomatic.

1. Centro de Estudos de Infectologia Pediátrica do Hospital Couto Maia da Secretaria de Saúde do Estado da Bahia Departamento de Pediatria da Faculdade de Medicina da Universidade Federal da Bahia. 2. Disciplina de Doenças Infecciosas e Parasitárias da Faculdade de Medicina da Universidade Federal da Bahia. 3. Instituto de Ciências da Saúde da Universidade Federal da Bahia, Salvador, BA.

Endereço para correspondência: Dr. Hagamenon R. da Silva. R. Humberto de Campos 159/301, Graça, 40150-130 Salvador, BA

Telefax: $5571247-5559$

e-mail: hagars@ufba.br

Recebido para publicação em 17/5/2001. 
A leptospirose é antropozoonose difundida por todo o mundo, exceto nas regiões polares, e com maior incidência nas regiões tropicais ${ }^{15}{ }^{12}$. O agente etiológico da leptospirose é a espiroqueta do gênero Leptospira, que compreende duas espécies: L. interrogans, patogênica para o homem e a $L$. biflexa, que é saprófita ${ }^{1314}$. A espécie $L$. interrogans tem vários sorovars ${ }^{27}$ e, no Brasil, o sorovar icterohemorragiae foi o mais identificado como agente causal em mais de $50 \%$ dos casos de Recife ${ }^{36}$, Salvador ${ }^{4}$ e São Paulo ${ }^{36}$.

A transmissão se dá, fundamentalmente, pelo contato com a água contaminada por espiroquetas, eliminadas através da urina de muitos animais, principalmente os ratos no meio urbano ${ }^{56912}$. A infecção também ocorre acidentalmente, em laboratório ou através da inoculação direta pela mordedura de ratos $^{1}$. Na criança, de modo geral, a leptospirose tem caráter

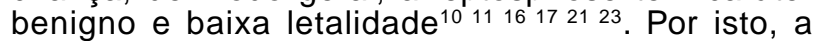
leptospirose-doença na criança é pouco relatada na literatura, apesar dela ter forte atração pela água na natureza e cujo contato é reconhecidamente fator de risco de desenvolvimento da doença. Por isto o objetivo principal deste trabalho foi diagnosticar a leptospiroseinfecção, aguda ou recente, na sua forma ambulatorial em crianças e adolescentes residentes nos domicílios de pacientes com leptospirose internados no Hospital Couto Maia em Salvador, BA; o objetivo secundário foi identificar manifestações clínicas neste grupo etário e avaliar fatores de riscos de contrair esta infecção.

\section{MATERIAL E MÉTODOS}

Este estudo seccional foi realizado no Hospital Couto Maia (HCMaia) em Salvador, referência para doenças infecciosas da Secretaria de Saúde do Estado da Bahia (SESAB). A população de referência foi a de residentes na região metropolitana de Salvador. Os casos-índices foram os pacientes internados entre abril e julho(período chuvoso) de 1993 no HCMaia com o diagnóstico de leptospirose, selecionados aleatoriamente e a população do estudo foi constituída pelas, crianças residentes (contactantes-domiciliares) nos domicílios dos pacientes com leptospirose (casos-índices).

Os critérios de inclusão foram: casos-índices, pacientes de qualquer idade ou sexo, internados no Hospital Couto Maia, com diagnóstico confirmado de leptospirose pelo método de Enzyme-linked immunosorbent assay (ELISA); contactantesdomiciliares (população de estudo), crianças de ambos sexos, idade entre 2 e 15 anos inclusive e concordância dos pais ou responsáveis legais de participação no estudo (termo de consentimento informado).

ELISA: de cada contactante-domiciliar foram coletadas amostras de sangue ( 3 a $5 \mathrm{ml}$ ) com intervalo médio de 20 dias. Os respectivos soros foram utilizados para pesquisa de anticorpos antileptospira, das classes $\operatorname{lgM}$ e $\operatorname{lgG}$, pelo método de ELISA ${ }^{26}$. Os ensaios tiveram 3 controles positivos e 3 controles negativos e todos os soros foram testados em duplicata. Para a realização do teste foi utilizada placa de microtitulação com 96 poços sensibilizados com extrato antigênico de L. interrogans, serovar icterohaemorragiae, cultivada no laboratório de Microbiologia do Instituto de Ciências da Saúde-UFBA, obtido por sonicação, na concentração de $10 \mu \mathrm{g} / \mathrm{ml}$ em tampão carbonato-bicarbonato $0,1 \mathrm{M}$,
$\mathrm{pH}$ 9,6. O bloqueio foi feito com soro - albumina bovina (BSA) a $2 \%$ em solução salina tamponada (PBS) 0,15M, pH 7,2 por duas horas à temperatura ambiente. Em cada poço foram colocados 100 $\mu$ l do soro diluído a 1:100 em PBS-BSA com $0,05 \%$ de Tween-20 e incubados por 1 hora a $37^{\circ} \mathrm{C}$. Após lavagens (6 vezes com PBS-Tween-20) foram adicionados $100 \mu l$ dos conjugados anti-IgM ou anti-IgG humanas (Sigma ) diluídos a 1:2000 em PBS-Tween-BSA e incubados por 1 hora a $37^{\circ} \mathrm{C}$. Os soros testados para IgM foram previamente tratados com solução absorvente de fator reumatóide (Behring). Após lavagens adicionou-se 100 $\mu$ l de solução substrato-cromógeno $\left(\mathrm{H}_{2} \mathrm{O}_{2}\right.$ - tetrametilbenzidina da Behring) e incubou-se por 30 minutos, no escuro, à temperatura ambiente. A reação foi interrompida com $\mathrm{H}_{2} \mathrm{SO}_{4}$ a $1 \mathrm{~N}$. Procedeu-se a leitura fotocolorimétrica com filtro de 450nm. Os soros testados foram considerados positivos para IgM e/ou lgG quando a leitura fotocolorimétrica ultrapassou a média dos títulos dos soros controles-negativos, desde que acima de dois valores do desvio-padrão (valores do cut-off ): 0,295 $\pm 0,049$ para lgM e 0,311 $\pm 0,064$ para lgG.

Os contactantes-domiciliares foram avaliados quanto as suas características demográficas, epidemiológicas, relação de parentesco com os casos-índices e manifestações clínicas, usando-se questionário-padrão quando da $1^{\text {a }}$ coleta de sangue, no ambulatório do HCMaia e reavaliados quando da $2^{\underline{a}}$ coleta de amostra sangüínea no $20^{\circ}$ dia, ou após este período durante a visita domiciliar para coleta da segunda amostra de sangue.

A análise estatística foi realizada através do programa EPI-INFO, versão 6.01b. As diferenças observadas nos testes estatísticos, paramétricos e não-paramétricos, foram consideradas significantes quando a probabilidade $(p)$ do erro 1 foi menor ou igual a 0,05 (5\%).

\section{RESULTADOS}

De 232 pacientes internados, no período do estudo com suspeita de leptospirose, foram selecionados 49 indivíduos e 25 desses preencheram os critérios de seleção dos casos-índices. Nos domicílios dos 25 casosíndices residiam 148 pessoas, porém somente $56,1 \%$ $(n=83)$ atenderam os critérios de inclusão dos contactantes-domiciliares. Desses contactantesdomiciliares, dez $(12 \%)$ foram excluídos posteriormente, porque a segunda amostra de sangue para os exames sorológicos (ELISA; IgG e IgM) não foi coletada. Portanto, o estudo foi realizado com 73 (88\%) dos contactantes-domiciliares. 
A Tabela 1 mostra a freqüência das variáveis demográficas dos casos-índices e dos contactantes domiciliares. O sexo masculino predominou em $96 \%$ $(24 / 25)$ dos casos e em $44 \%(11 / 25)$ a idade foi inferior a 15 anos (limites de 2 a 15 anos). Entre os contactantes-domiciliares $(n=73)$, não houve predomínio de nenhum dos sexos, tanto nos da faixa etária de 2 a 8 anos como na de 9 a 15 anos.

Os resultados do (ELISA), antileptospira (IgM e lgG), na Tabela 2, classificaram as crianças estudadas ( $n=73$ ) em três grupos sorológicos: 1) grupo A com infecção aguda ou recente, IgM-positiva pelo ELISA - na primeira,

Tabela 1 - Freqüências das variáveis demográficas dos casos-índices, pacientes portadores de leptospirose e de crianças, contactantesdomiciliares, procedentes de Salvador, Bahia.

\begin{tabular}{|c|c|c|c|c|}
\hline \multirow[b]{2}{*}{ Variável } & \multicolumn{2}{|c|}{$\begin{array}{c}\text { casos-índices } \\
(\mathrm{n}=25) \\
\end{array}$} & \multicolumn{2}{|c|}{$\begin{array}{l}\text { contactantes-domiciliares } \\
\qquad(\mathrm{n}=73)\end{array}$} \\
\hline & $\mathrm{n}$ & $\%$ & $\mathrm{n}$ & $\%$ \\
\hline \multicolumn{5}{|l|}{ Sexo } \\
\hline masculino & 24 & 96 & 39 & 53,4 \\
\hline feminino & 1 & 4 & 34 & 46,6 \\
\hline \multicolumn{5}{|l|}{ Raça } \\
\hline branca & - & - & 5 & 6,9 \\
\hline negra & - & - & 52 & 71,2 \\
\hline mulata & - & - & 16 & 21,9 \\
\hline Idade média (anos) & \multicolumn{2}{|c|}{$24,4( \pm 15,9)$} & \multicolumn{2}{|c|}{$24,4( \pm 15,9)$} \\
\hline
\end{tabular}

Tabela 2 - Análise das variáveis pesquisadas nas crianças dos grupos sorológicos A (leptospirose), B (não-infectado) e C (infecção passada) de Salvador, Bahia.

\begin{tabular}{|c|c|c|c|c|c|c|c|c|c|}
\hline \multicolumn{10}{|l|}{ Grupos sorológicos } \\
\hline \multirow[t]{2}{*}{ Variável } & \multicolumn{2}{|c|}{$A(n=30)$} & \multicolumn{2}{|c|}{$B(n=34)$} & \multicolumn{2}{|c|}{$C(n=9)$} & \multicolumn{2}{|c|}{ Total } & \multirow{2}{*}{$\begin{array}{r}\text { A vs } B \\
p\end{array}$} \\
\hline & $\mathrm{n}$ & $\%$ & $\mathrm{n}$ & $\%$ & $n$ & $\%$ & $n$ & $\%$ & \\
\hline \multicolumn{10}{|l|}{ Sexo } \\
\hline masculino & 12 & 30,7 & 20 & 51,2 & 7 & 18,1 & 39 & 53,4 & 0,13 \\
\hline feminino & 18 & 52,9 & 14 & 41,1 & 2 & 6,0 & 34 & 46,6 & \\
\hline \multicolumn{10}{|l|}{ Raça } \\
\hline branca & 2 & 40,0 & 3 & 60,0 & - & - & 5 & 6,8 & 0,93 \\
\hline mulata & 18 & 60,0 & 25 & 48,1 & 9 & 17,3 & 52 & 71,2 & \\
\hline negra & 10 & 62,3 & 6 & 37,7 & - & - & 16 & 22,0 & \\
\hline \multicolumn{10}{|l|}{ Idade (anos) } \\
\hline $2-8$ & 10 & 29,4 & 19 & 55,8 & 5 & 14,8 & 34 & 46,6 & 0,07 \\
\hline $9-15$ & 20 & 51,2 & 15 & 28,4 & 4 & 10,4 & 39 & 53,4 & \\
\hline média $\pm d p$ & \multicolumn{2}{|c|}{$9,8 \pm 3,0$} & \multicolumn{2}{|c|}{$7,9 \pm 3,0$} & \multicolumn{2}{|c|}{$8,5 \pm 3,7$} & & & \\
\hline \multicolumn{10}{|l|}{ Parentesco c/ caso índice } \\
\hline irmão & 15 & 51,7 & 11 & 37,9 & 3 & 10,4 & 29 & 39,7 & 0,005 \\
\hline filho & 4 & 16,0 & 18 & 72,0 & 3 & 12,0 & 25 & 34,2 & \\
\hline outros & 11 & 57,8 & 5 & 26,3 & 3 & 15,9 & 19 & 26,1 & \\
\hline \multicolumn{10}{|l|}{$\begin{array}{l}\text { Contato com a água } \\
\text { (fonte de contágio) }\end{array}$} \\
\hline $\operatorname{sim}$ & 18 & 52,9 & 11 & 32,3 & 5 & 14,8 & 34 & 46,6 & 0,02 \\
\hline não & 12 & 30,7 & 23 & 58,9 & 4 & 10,4 & 39 & 53,4 & \\
\hline \multicolumn{10}{|l|}{$\begin{array}{l}\text { Presença de cão } \\
\text { no domicílio }\end{array}$} \\
\hline $\operatorname{sim}$ & 12 & 36,3 & 15 & 45,4 & 6 & 18,3 & 33 & 45,2 & 0,74 \\
\hline não & 18 & 45,0 & 19 & 47,5 & 3 & 7,5 & 40 & 54,8 & \\
\hline \multicolumn{10}{|l|}{ Procedência } \\
\hline subúrbio-ferroviário* & 10 & 35,7 & 12 & 42,8 & 6 & 21,5 & 28 & 8,3 & 0,71 \\
\hline Outros & 20 & 44,4 & 22 & 48,8 & 3 & 6,8 & 45 & 61,7 & \\
\hline
\end{tabular}

* Um terço(10/20) dos casos do grupo A e dois terço (6/3) do grupo C procederam do distrito sanitário subúrbio ferroviário dos 12 que compõem a região metropolitana de Salvador. 
na segunda ou em ambas as amostras séricas, correspondendo a 41,1\% (30/73) dos casos; 2) grupo B com $34(46,6 \%)$ crianças sem infecção anterior ou recente (IgM e lgG negativas, nas duas amostras séricas) e 3) grupo $\mathrm{C}$ com nove (12,3\%) crianças, portadoras de lgG-positiva e títulos compatíveis, em ambas as amostras séricas, com diagnóstico de infecção antiga.

Todos os casos-índices e contactantes-domiciliares referiram presença de ratos no seus domicílios ou peridomicílios. As características demográficas e epidemiológicas e a relação de parentesco com os casos-índices, dos contactos domiciliares foram apresentadas (Tabela 2) sendo que as do grupo $A$ (leptospirose-infecção ou doença) foram comparadas com as do grupo B (não-infectadas). As distribuições pelos grupos racias $(p>0,93)$ e por sexo $(p>0,13)$ foram semelhantes,contudo houve tendência de predomínio do sexo feminino no grupo A e do masculino no grupo B. Apesar da freqüência maior $(51,2 \%)$ de crianças do grupo A com 9 a 15 anos de idade, a distribuição intervalar ficou no limite de significância estatística $\left(\chi^{2}=3,27, p>0,07\right)$ e as médias das idades foram semelhantes $(t=0,53, p>0,50)$. parentesco dos 64 contactantes-domiciliares, dos grupos A e B, (Tabela 2) foram relacionados com os respectivos casos-índices com a seguinte distribuição geral: 22 eram filhos, 26 irmãos e 16 tinham outros parentescos ou eram moradores sem ligação consangüínea. Esta distribuição foi desigual $\left(\chi^{2}=11,4\right.$, $\mathrm{p}<0,005)$, porque predominaram como casos-índices, no grupo A os irmãos (51,7\%) e no grupo B os filhos (72\%).

O contato recente com água na natureza, como provável fonte de contágio, foi referido por 18 (52,9\%) dos casos do grupo A e 11 (32,3\%) dos do grupo B, sendo a diferença estatisticamente significante $\left(\chi^{2}=4,92, p=0,02\right)$. A presença de cão no domicílio teve distribuição semelhante nos dois grupos $(p=0,74)$. Mais de um terço $(35,7 \%)$ dos casos do grupo A residia em um só Distrito Sanitário (Subúrbio Ferroviário) dos 12 do município de Salvador. No grupo C (infecção passada) 21,5\% foi procedente deste mesmo subúrbio.

A associação das variáveis idade e sexo, na Tabela 3, mostra que entre as IgM-positiva (grupo A), da faixa etária de 9 a 15 anos, predominaram $\left(\chi^{2}=4,64, p=0,03\right)$ as do sexo feminino (70\%), enquanto nas do grupo $B$ (IgM-negativa) foram mais freqüentes as crianças do

Tabela 3 - Distribuição dos contactantes domiciliares dos grupos A (leptospirose) e B ( não infectado) segundo faixa etária e sexo.

\begin{tabular}{|c|c|c|c|c|c|c|c|c|c|c|c|c|}
\hline \multirow{3}{*}{$\begin{array}{l}\text { Sexo } \\
\text { Idade (anos) }\end{array}$} & \multicolumn{4}{|c|}{$\begin{array}{c}\text { Masculino } \\
\text { Grupos }\end{array}$} & \multicolumn{4}{|c|}{$\begin{array}{c}\text { Feminino } \\
\text { Grupos }\end{array}$} & & & \multirow{3}{*}{$\chi^{2}$} & \multirow{3}{*}{$p$} \\
\hline & \multicolumn{2}{|c|}{ A } & \multicolumn{2}{|c|}{ B } & \multicolumn{2}{|c|}{ A } & \multicolumn{2}{|c|}{$B$} & \multicolumn{2}{|c|}{ Total } & & \\
\hline & $\mathrm{n}$ & $\%$ & $\mathrm{n}$ & $\%$ & $\mathrm{n}$ & $\%$ & $\mathrm{n}$ & $\%$ & $\mathrm{n}$ & $\%$ & & \\
\hline $2-8$ & 6 & 60,0 & 10 & 52,6 & 4 & 40,0 & 9 & 47,4 & 29 & 45,3 & 0,14 & 0,70 \\
\hline $9-15$ & 6 & 30,0 & 10 & 66,7 & 14 & 70,0 & 5 & 33,3 & 35 & 54,7 & 4,64 & 0,03 \\
\hline Total & 12 & 18,7 & 20 & 31,2 & 18 & 28,1 & 14 & 12,0 & 64 & 100,0 & & \\
\hline
\end{tabular}

sexo masculino (66,7\%); enquanto na faixa de 2 a 8 anos, a distribuição por sexo foi semelhante $(p=0,70)$.

$\mathrm{Na}$ Tabela 4, os casos dos grupos A e B foram distribuídos conforme a idade dos seus casos-índices. A taxa de risco de aquisição da infecção para os contatos-domiciliares de casos-índices com 15 ou menos anos de idade foi de 3,76 vezes maior do que os contactantes de casos-índices com mais de 15 anos de idade, sendo a diferença estatisticamente significante $(p<0,02)$.

\begin{tabular}{|c|c|c|c|c|c|c|}
\hline \multirow{3}{*}{$\begin{array}{l}\text { Caso-índice } \\
\text { idade (anos) }\end{array}$} & \multicolumn{4}{|c|}{ Contactantes-domiciliares } & & \\
\hline & \multicolumn{2}{|c|}{ Grupo A } & \multicolumn{2}{|c|}{ Grupo B } & \multicolumn{2}{|c|}{ Total } \\
\hline & $\mathrm{n}^{\circ}$ & $\%$ & $\mathrm{n}^{\circ}$ & $\%$ & $\mathrm{n}^{\circ}$ & $\%$ \\
\hline$\leq 15$ & 20 & 62,5 & 12 & 37,5 & 32 & 50,0 \\
\hline$>15$ & 10 & 31,3 & 22 & 68,7 & 32 & 50,0 \\
\hline Total & $30 a$ & 46,8 & 34 & 53,2 & 64 & 100,0 \\
\hline
\end{tabular}

A Tabela 5 mostra a carência do serviço público de coleta de lixo nas áreas de procedência dos casos do grupo A $(93,3 \%)$ e do B $(32,4 \%)$, com diferença altamente significativa ( $p=0,0000006)$.

Quanto às manifestações clínicas, (dados não tabelados), das 30 crianças do grupo A com infecção aguda ou recente, apenas cinco (16,7\%), todas do sexo feminino apresentaram alterações mórbidas recentes. Os sintomas referidos eram típicos de resfriado comum: coriza, tosse e dores musculares. Apenas três destas crianças apresentaram febre e uma delas a queixa predominante foi dores intensas nas panturrilhas. A 
maioria (83,3\%) das crianças do grupo A (25/30) não relatou nenhuma manifestação clínica. Entre as crianças ( $n=34$ ) do grupo B (não-infectadas), 14,7\% (5/34) também referiram manifestações clínicas recentes, sendo $8,8 \%$ $(3 / 34)$ com síndrome gripal, semelhantes às do grupo $A$ e $5,9 \%(2 / 34)$ com sintomas gastro-intestinais (diarréia e vômitos). Entretanto, ao aplicar o teste exato de Fischer, não se observou diferença estatística $(p=0,28)$ entre os casos do grupo A (5/30) com síndrome gripal e os do grupo B (3/34) com quadro clínico semelhante. Entre as nove crianças do grupo $\mathrm{C}$, (infecção passada), não se identificou de história pregressa de leptospirose.

Tabela 5 - Frequência do serviço público de limpeza urbana nas áreas de procedência dos contactantes domiciliares dos grupos $A$ e $B$ em Salvador, Bahia.

\begin{tabular}{|c|c|c|c|c|c|c|}
\hline \multirow[b]{3}{*}{ Grupo } & \multicolumn{6}{|c|}{ Serviço Público de coleta de lixo } \\
\hline & \multicolumn{2}{|c|}{$\operatorname{sim}$} & \multicolumn{2}{|c|}{ não } & \multicolumn{2}{|c|}{ total } \\
\hline & $\mathrm{n}^{\circ}$ & $\%$ & $\mathrm{n}^{\circ}$ & $\%$ & $\mathrm{n}^{\circ}$ & $\%$ \\
\hline $\bar{A}$ & 2 & 6,7 & 28 & 93,3 & 30 & 46,8 \\
\hline B & 23 & 67,6 & 11 & 32,4 & 34 & 53,2 \\
\hline Total & 25 & 39,1 & 39 & 60,9 & 64 & 100,0 \\
\hline
\end{tabular}

\section{DISCUSSÃO}

O Hospital Couto Maia (HCMaia) é a instituição de referência, da rede pública de Salvador no Estado da Bahia, para internação de pacientes portadores de doenças infecciosas e parasitárias e, especialmente, os casos clínicos suspeitos de leptospirose ${ }^{1819}$. Na nossa casuística, das 73 crianças da faixa etária de dois a 15 anos contactantes domiciliares de pacientes internados com leptospirose naquele Hospital, 30 (41,1\%) tiveram infecção aguda ou recente por $L$. interrogans e cinco $(16,7 \%)$ referiram sintomatologia semelhante ao resfriado comum. Alguns autores ${ }^{58}$, em inquéritos soroepidemiológicos, observaram soro-positividade crescente com o aumento da idade da população de estudo. Como não havia referência à forma clínica clássica da leptospirose estes autore ${ }^{58}$ concluíram que as formas clínicas assintomáticas e oligossintomáticas seriam as apresentações mais comuns na infância.

$\mathrm{O}$ encontro de IgM positiva em 30 contactantesdomiciliares, de pacientes internados no HCMaia, sendo $25(83,3 \%)$ casos de infecção assintomática e cinco $(16,7 \%)$ com síndrome gripal, classificados na forma ambulatorial oligossintomática, confirma a benignidade da leptospirose na infância, apesar de ter havido freqüência semelhante da síndrome gripal entre as crianças do grupo B (não-infectados).

Nas crianças do grupo $C$, também não houve referência de antecedentes de manifestações clínicas de doença compatível com leptospirose, reforça mais ainda a freqüência elevada das formas subclínicas desta infecção. A leptospirose na criança pode também se apresentar sob outras formas clínicas ainda pouco estudadas $^{22} 24$ ou ser adquirida intra-útero ${ }^{20}$.

Assim é que a forma meníngea da leptospirose ${ }^{2}$ passa habitualmente despercebido ${ }^{22}$. No HCMaia, Costa e cols ${ }^{7}$ encontraram cinco $(31,2 \%)$ de leptospirose em 16 casos de síndrome de meningite asséptica. Em Morón - Cuba, Suarez Hernandez e cols ${ }^{24}$ descreveram a leptospirose em seis crianças após banho em rio, e quatro destes casos tiveram síndrome de meningite asséptica causada pelo sorovar canicola. Em outra investigação, Suarez Hernandez e cols ${ }^{25}$, em 157 casos de síndrome de meningite asséptica pelo teste da microaglutinação, encontraram em 16 (10,2\%) a L. interrogans como agente causal.

A infecção pela leptospira no período neonatal é rara e ocorre por transmissão vertical ${ }^{20}$. Shaked e cols descreveram um caso por transmissão via transplacentária e também revisaram outros 15 casos publicados, diagnosticados por meio do exame da placenta ou do recém-nascido. Destes 15, três neonatos tiveram a forma congênita, foram tratados com penicilina e apresentaram boa evolução clínica ${ }^{20}$.

Cruz $^{8}$ encontrou $17,4 \%(n=254)$ de soro-positividade (IgG para leptospira), pelo teste de micro-aglutinação, em estudo envolvendo 1458 escolares de seis a 12 anos de idade, de São João do Meriti, Rio de Janeiro. No presente estudo o diagnóstico de infecção-antiga foi de 12,3\% (grupo C), freqüência próxima a observada por aquele pesquisador ${ }^{8}$. Em Baltimore-USA, Childs e cols ${ }^{5}$, em estudo semelhante e utilizando o teste de ELISA (IgG), encontraram $16,1 \%$ das crianças com sorologia positiva (185/1150), porém, estes autores ${ }^{5}$ não relataram a infecção distribuída por faixa etária. No presente estudo, a elevada freqüência $(41,1 \%)$ de leptospirose ocorreu porque ele foi desenvolvido durante o período chuvoso, de maior incidência da leptospirose na população da cidade do Salvador ${ }^{3}$.

Quando se analisou a distribuição da infecção (grupo A), aguda ou recente, por sexo, houve o predomínio do feminino, à semelhança do observado por $\mathrm{Cruz}^{8}$, contudo, em ambos os estudos a diferença não alcançou significância estatística. Por outro lado, a associação da idade ao sexo, neste estudo, mostrou que as meninas, na faixa etária de nove a 15 anos, tiveram freqüência maior de sorologia positiva para lgM antileptospira (infecção aguda ou recente). A maior 
ocorrência de infecção subclínica no sexo feminino contrapôs-se à maioria das formas sintomáticas clássicas hospitalares que ocorrem no sexo masculino ${ }^{21}$. É provável que isto seja decorrente da duração maior da exposição às fontes de infecção das crianças do sexo masculino, com possibilidade de serem infectados com maior carga infectante.

A probabilidade do contactante-domiciliar contrair a infecção, devido ao seu grau de parentesco com o casoíndice, foi maior quando o irmão era o caso-índice. Isto deveu-se possivelmente por terem fonte de infecção comum e hábitos e/ou costumes mais semelhantes, do que os observados em outros tipos de parentesco e também, porque nas crianças do grupo $A, 62,5 \%$ dos casos estavam vinculados a casos-índices de idade inferior a 15 anos. Este achado reforça a ideia que estes casos tenham tido como fonte de infecção, a mesma do caso-índice.

A freqüência do serviço público de coleta do lixo em Salvador foi a variável mais determinante do número de indivíduos do grupo A (IgM-positivos). Isto porque o lixo acumulado favorece o aumento da população murina. Assim, naquelas áreas com serviços públicos de limpeza urbana precários, é possível que a elevação da população de roedores (Rattus $s p$ ) aumentou a probabilidade de transmissão da $L$. interrogans, especialmente no distrito do Subúrbio Ferroviário da cidade do Salvador, onde há elevado déficit de coleta de lixo e também de onde provem a maior parte dos pacientes de leptospirose ${ }^{34}$.

Em conclusão, as apresentações clínicas atípicas ou benignas da infecção pela $L$. interrogans na criança passam despercebidas de modo que, a freqüência da doença neste grupo etário é subestimada. Estes achados evidenciam a necessidade de estudo longitudinal, com o objetivo de: 1 estimar a incidência da infecção e da leptospirose clínica na faixa etária pediátrica; 2 analisar os fatores de risco, demográficos, epidemiológicos e 3 . determinar o espectro clínico das formas oligossintomáticas.

\section{REFERÊNCIAS BIBLIOGRÁFICAS}

1. Barbosa LT. Espiroquetose ictero-hemorragica em lactente de um mês. Jornal de Pediatria 9: 2-14, 1943.

2. Bittencourt JMT, Fujioka T, Corrêa MOAB, Tranchesi J, Bedrikow B. O líquido céfalo raquidiano na moléstia de Weil. Revista do Instituto Adolfo Lutz 12: 145-161, 1952.

3. Caldas EM. Leptospirose na cidade de Salvador. Estudo epidemiológico, com alguns aspectos sológicos, clínicos e laboratoriais. Tese de Mestrado, Universidade Federal da Bahia, Salvador, BA, 1976.

4. Caldas EM, Costa E, Sampaio MB. Leptospirose na cidade do Salvador (Brasil). Alguns aspectos clínicos e laboratoriais. Revista do Instituto de Medicina Tropical de São Paulo 20: 164-176, 1978.

5. Childs JE, Schwartz BS, Ksiazek TG, Graham RR, Leduc JW, Glass Gek. Risk factors associated with antibodies to leptospiras in inner-city residents of Baltimore: a protective role for cats. American Journal of Public Health 82: 597-599, 1992.

6. Corrêa MOA. Human leptospirosis in Brazil. International Journal Zoonosis 2: 1-9, 1975.

7. Costa E, Costa Y, Santos RR, Silva HR da, Silva NG, Silva IC, Miranda Filho GV, Caldas EM, Sampaio EB. Leptospirose: uma causa de meningite asséptica. Revista Médica da Bahia 23: 107108, 1977.

8. Cruz MLS. O inquérito para prevalência e fatores de risco da leptospirose em escolares de São João de Meriti, Rio de Janeiro. Tese de Mestrado, Instituto Oswaldo Cruz, Rio de Janeiro, RJ, 1992.

9. Detmers RY, Frank RR, Thieman AB, Dekmers PA. Exposure to Leptospira icterohemorrhagiae in inner-city and suburban. Journal Family Practice 17: 1007/1111, 1983.

10. Everard COR, Haynes RJ, Edwards CN. Leptospiral Infection in School-children from Trinidad and Barbados. Epidemiology and Infection 103: 143-156, 1989.

11. Everard COR, Maude GH, Hayes RJ. Leptospiral infection: a household serosurvey in urban and rural communities in Barbados and Trinidad. Annals of Tropical Medicine and Parasitology 84: 255-266, 1990.
12. Faine S (ed) Guidelines for the control of leptospirosis. World Health Organization, (WHO Offset Publication n. 67), Geneva, 1982.

13. Farrar WE. Leptospira species (leptospirosis). In: Mandell GL, Douglas RG, Bennett JE (eds.) Principles and practice of Infectious diseases. $4^{\text {th }}$ edition, Churchill Livingstone, New York, v2, p. 2137-2141, 1995.

14. Feigin RD, Anderson DC. Leptospirosis. In: Feigin RD, Cherry JD (eds) Textbook of pediatric infectious diseases. Saunders, Philadelphia, p.1167-1181, 1992.

15. Fundação Nacional de Saúde. Programa Nacional de Controle de Leptospirose. Proposta de ações de controle de leptospirose a nível nacional. CENEPI/Ministério da Saúde, Brasília, 1993.

16. Galvão PAA, Scheinberg MA, Pereira Junior W, Fucs M, SonneWend JPAS, Farhat CK, Corrêa MOA. Leptospirose na infância. Pediatria Prática 39: 45-50, 1968.

17. Lombardi F. Considerações sobre alguns casos de icterícia leptospirilar na infância. Revista Brasileira de Medicina 2: 633-636, 1945.

18. Machado R, Costa E. Leptospirose em Salvador (1970-1975). Alguns aspectos clínicos e epidemiológicos. Revista Médica da Bahia 23: 194-207, 1977.

19. Secretaria da Saúde do Estado da Bahia. Leptospirose. Boletim Epidemiológico/SESAB, 2:1-7, 1988.

20. Shaked Y, Shpilberg O, Samra D, Samra Y. Leptospirosis in pregnancy and its effect on the fetus: case report and review. Clinical Infectious Diseases 17: 241-253, 1993.

21. Silva HR, Matos A, Queiroz I, Campos GS. Leptospirose em pediatria: estudo clínico de 23 casos no Hospital Couto Maia. In: Resumos do VIII Congresso Brasileiro de Infectologia Pediátrica, Salvador, BA, p.47,1992.

22. Sperber SJ, Schleupner CJ. Leptospirosis: a forgotten cause of aseptic meningitis and multisystem febrile illness. Southern Medical Journal 82: 1285-1287, 1989.

23. Steigman AJ. Leptospirose. In: Nelson WE, Vaughan VC, Mckay RJ (eds) Tratado de Pediatria. Salvat, Barcelona, p. 629-630, 1990. 
24. Suarez Hernandez M, Bustelo Aguila J, Perez Gonzalez L, Gorgoy Gonzalez V. Brote de leptospirosis en niños con predomino meningoencefalico, en el municipio Morón. Revista Cubana de Medicina Tropical 43: 136-139, 1991.

25. Suarez Hernandez M, Perez de Corcho M, Bal Llovera G, Arias Riquesne J. Estudio clinico-epidemiologico de casos de meningoencefalitis por leptospira en la Provincia de Ciego de Avila. Revista Cubana de Higiene y Epidemiologia 27: 37-48, 1989.
26. Terpstra WJ, Ligthart GS, Schoone GJ. Serodiagnosis of human leptospirosis by enzyme-linked immunosorbent assay (ELISA). Zentralblatt für Bakteriologie 247: 400-405, 1980.

27. Yasuda PH, Steigerwalt AG, Sulzer KR, Kaufmann AF, Rogers F, Brenner DJ. Deoxyribonucleic acid relatedness between serogroups and serovars in the family leptospiraceae with proposals for seven new leptospira species. International Journal of Systematic Bacteriology 37: 407-415, 1987. 